\title{
Corrigendum
}

\section{Corrigendum to "Incorporating Family Function into Chronic Pain Disability: The Role of Catastrophizing"}

\author{
Fatemeh Akbari, ${ }^{1}$ Mohsen Dehghani, ${ }^{2}$ Ali Khatibi, ${ }^{3,4}$ and Tine Vervoort ${ }^{5}$ \\ ${ }^{1}$ Family Research Institute, Shahid Beheshti University, G.C., Tehran 1983963113, Iran \\ ${ }^{2}$ Department of Psychology, Shahid Beheshti University, G.C., Tehran 1983963113, Iran \\ ${ }^{3}$ Psychology Department, Bilkent University, 06800 Ankara, Turkey \\ ${ }^{4}$ Interdisciplinary Program in Neuroscience, Bilkent University, 06800 Ankara, Turkey \\ ${ }^{5}$ Department of Experimental-Clinical and Health Psychology, Ghent University, 9000 Ghent, Belgium
}

Correspondence should be addressed to Fatemeh Akbari; fatemeh.akbari1394@gmail.com

Received 18 June 2017; Accepted 22 June 2017; Published 25 October 2017

Copyright (C) 2017 Fatemeh Akbari et al. This is an open access article distributed under the Creative Commons Attribution License, which permits unrestricted use, distribution, and reproduction in any medium, provided the original work is properly cited.

In the article titled "Incorporating Family Function into Chronic Pain Disability: The Role of Catastrophizing" [1], an acknowledgment should be added as follows.

The authors would like to acknowledge Dr. Majtaba Habibi, a co-adviser of Dr. Akbari's thesis, for his role in the project and revising an earlier version of the manuscript.

This follows a decision by Shahid Beheshti University about an authorship claim by Dr. Habibi.

\section{References}

[1] F. Akbari, M. Dehghani, A. Khatibi, and T. Vervoort, "Incorporating family function into chronic pain disability: The role of catastrophizing," Pain Research and Management, vol. 2016, Article ID 6838596, 9 pages, 2016. 


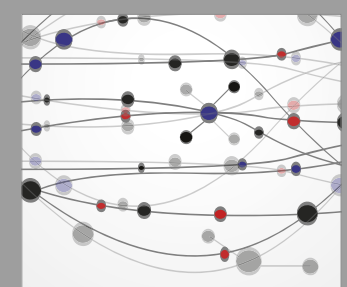

The Scientific World Journal
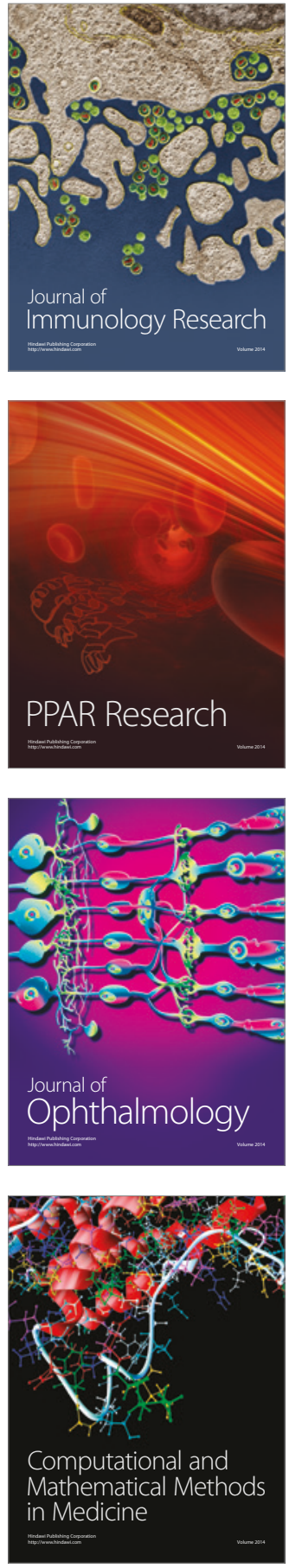

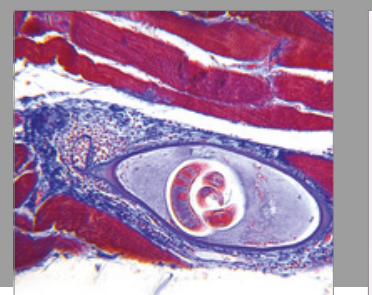

Gastroenterology Research and Practice
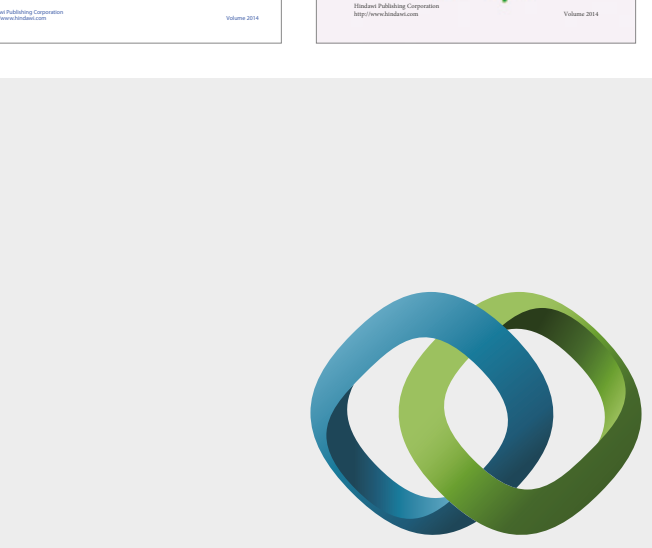

\section{Hindawi}

Submit your manuscripts at

https://www.hindawi.com
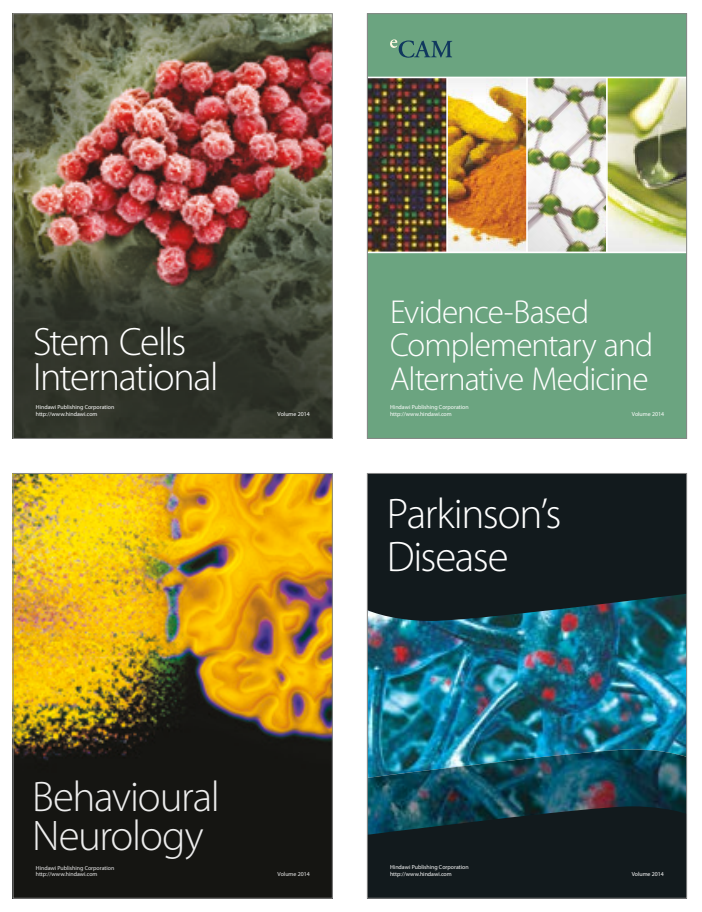
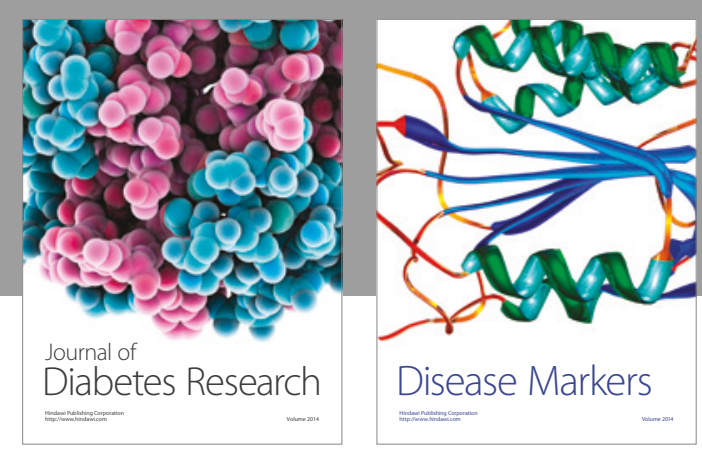

Disease Markers
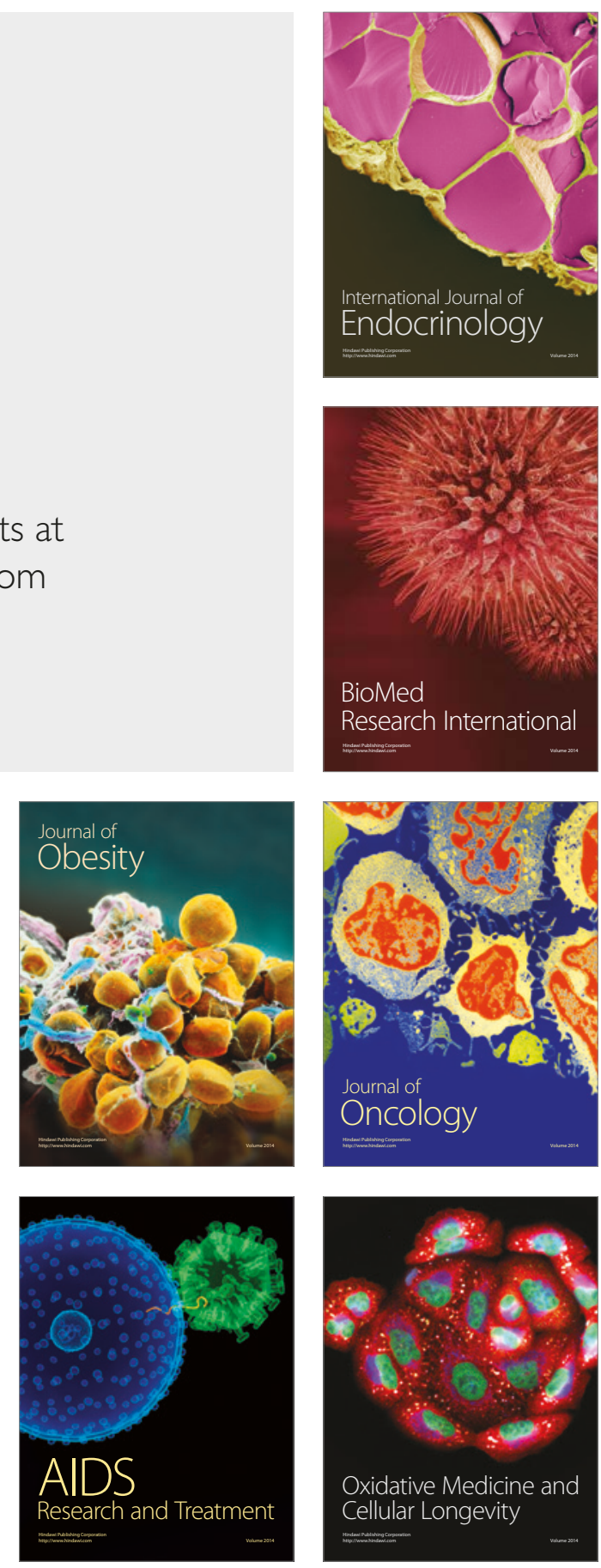